\title{
Análise temporal e epidemiológica da coinfecção Tuberculose-HIV no Estado do Pará, 2010-2020
}

\author{
Temporal and epidemiological analysis of Tuberculosis-HIV coinfection in the State of \\ Pará, 2010-2020
}
Análisis temporal y epidemiológico de la coinfección Tuberculosis-VIH en el Estado de Pará, 2010-2020

Taynara da Costa Silva ${ }^{1}$, Cássia Vitória Passos dos Santos ${ }^{1}$, Thayana de Almeida Vieira ${ }^{1}$, Matheus de Oliveira Queiroz ${ }^{1}$, Karytta Sousa Naka1*.

\section{RESUMO}

Objetivo: Analisar a distribuição temporal e epidemiológica da coinfecção Tuberculose e Vírus da Imunodeficiência Adquirida (TB-HIV) no Estado do Pará, no período de 2010-2020. Métodos: Trata-se de um estudo ecológico, retrospectivo e descritivo, de séries temporais, dos casos confirmados de TB coinfectados com HIV obtidos no Sistema de Informação de Agravos de Notificação. Resultados: Foram registrados 48.684 casos de TB, dentre estes, 4.307 apresentaram sorologia anti-HIV positiva, caracterizando uma coinfecção TB-HIV de 8,8\% no estado do Pará no período estudado. O ano de 2019 apresentou maior número de casos, com 12,6\% e, Belém, foi o município mais acometido $(66,4 \%)$. Houve predominância de casos no sexo masculino $(70,3 \%)$, faixa etária entre 20 a 39 anos $(57,0 \%)$ e ensino médio completo $(41,9 \%)$. A forma clínica pulmonar $(68,3 \%)$, casos novos $(78,6 \%)$ e cura $(47,7 \%)$ apresentaram maior percentual. Em relação aos grupos vulneráveis, 233 casos foram confirmados, correspondendo a 5,4\% dos casos de coinfecção TBHIV. Conclusão: Os resultados presentes neste estudo fornecem informações epidemiológicas e ao longo do tempo sobre a coinfecção TB-HIV relevantes para futuras pesquisas e, também, favorecem a implementação de estratégias de prevenção e controle na população, visando melhor qualidade de vida aos indivíduos acometidos pela coinfecção.

Palavras-chave: Coinfecção pelo HIV, Epidemiologia, Estudos ecológicos.

\begin{abstract}
Objective: To analyze the temporal and epidemiological distribution of Tuberculosis and Acquired Immunodeficiency Virus (TB-HIV) coinfection in the State of Pará, in the period 2010-2020. Methods: This is an ecological, retrospective and descriptive study, of time series, of confirmed cases of TB co-infected with HIV obtained from the Notifiable Diseases Information System. Results: A total of 48,684 cases of TB were recorded, among which 4,307 had positive anti-HIV serology, characterizing a TB-HIV co-infection of $8.8 \%$ in the state of Pará during the period studied. The year 2019 had the highest number of cases, with $12.6 \%$, and Belém, as the most affected municipality (66.4\%). There was a predominance of cases in males $(70.3 \%)$, age group between 20 and 39 years (57.0\%) and complete high school (41.9\%). The pulmonary clinical form $(68.3 \%)$, new cases $(78.6 \%)$ and cure $(47.7 \%)$ had the highest percentage. Regarding vulnerable groups, 233 cases were confirmed, corresponding to $5.4 \%$ of TB-HIV co-infection cases. Conclusion: The results presented in this study provide epidemiological information and over time on TB-HIV coinfection relevant for future research and also favor the implementation of prevention and control strategies in the population, aiming at a better quality of life for individuals affected by the disease coinfection.
\end{abstract}

Keywords: Coinfection HIV, Epidemiology, Ecological studies.

${ }^{1}$ Faculdade Estácio de Castanhal, Castanhal - PA. *E-mail: karyttasousa@hotmail.com 


\section{RESUMEN}

Objetivo: Analizar la distribución temporal y epidemiológica de la coinfección Tuberculosis y Virus de la Inmunodeficiencia Adquirida (TB-VIH) en el Estado de Pará, en el período 2010-2020. Métodos: Se trata de un estudio ecológico, retrospectivo y descriptivo, de serie temporal, de casos confirmados de TB coinfectados por VIH obtenidos del Sistema de Información de Enfermedades de Declaración Obligatoria. Resultados: Se registraron un total de 48.684 casos de TB, entre los cuales 4.307 tenían serología anti-VIH positiva, caracterizando una coinfección TB-VIH del 8,8\% en el estado de Pará durante el período estudiado. El año 2019 tuvo el mayor número de casos, con $12,6 \%$, y Belém, como el municipio más afectado $(66,4 \%)$. Hubo predominio de casos en el sexo masculino (70,3\%), grupo de edad entre 20 y 39 años $(57,0 \%)$ y secundaria completa $(41,9 \%)$. La forma clínica pulmonar $(68,3 \%)$, casos nuevos $(78,6 \%)$ y curación $(47,7 \%)$ tuvieron el mayor porcentaje. En cuanto a los grupos vulnerables, se confirmaron 233 casos, que corresponden al 5,4\% de los casos de coinfección TB-VIH. Conclusión: Los resultados presentados en este estudio brindan información epidemiológica y en el tiempo sobre la coinfección TB-VIH relevante para futuras investigaciones y también favorecen la implementación de estrategias de prevención y control en la población, visando una mejor calidad de vida para las personas afectadas por la enfermedad coinfección.

Palabras clave: Coinfección por HIV, Epidemiología, Estudios ecológicos.

\section{INTRODUÇÃO}

A Tuberculose (TB), doença infectocontagiosa causada pela bactéria Mycobacterium tuberculosis, é uma das causas mais importantes de morte no mundo. Até o surgimento da pandemia do novo coronavírus (COVID-19), a TB foi a principal causa de morte de um único agente infeccioso, ultrapassando a Síndrome da Imunodeficiência Adquirida (Aids). Frequentemente, a TB é associada a uma enfermidade do passado, contudo, ainda existem importantes deficiências no diagnóstico e tratamento da infecção (MINISTÉRIO DA SAÚDE, 2021).

O comprometimento imunológico causado pela Aids impacta na multiplicação do $M$. tuberculosis e na probabilidade de adoecimento na presença do Vírus da Imunodeficiência Adquirida (HIV) e outras formas de imunodepressão (BARBOSA KMB, et al., 2020). Segundo Cavalin RF, et al. (2020) a infecção pelo HIV representa o mais importante fator de risco para a TB, estimando uma probabilidade de 19 vezes maior para os indivíduos infectados pelo HIV quando comparado à população geral.

O HIV/Aids tem um impacto significativo sobre o perfil epidemiológico e controle da endemia tuberculosa, uma vez que é a principal causa de morte entre as pessoas que convivem com a doença. $O$ risco de adoecimento é comum em todas as faixas etárias, sendo maior entre os menores de cinco anos, adolescentes, adultos jovens e idosos (BLACK TLP e CARDOSO MD, 2019). A TB e o HIV são infecções consideradas um problema de saúde pública à nível mundial e, portanto, a inter-relação entre as duas é um agravo que deve ser ainda mais discutido, em virtude dos impactos acarretados no alcance das metas estabelecidas pela Organização Mundial da Saúde (BALDAN SS, et al., 2017).

No Brasil, em 2019, foi observado um percentual de 10,3\% de casos novos de TB coinfectados pelo vírus HIV (TB-HIV) (MINISTÉRIO DA SAÚDE, 2021). Esse percentual ainda representa uma grave falha da saúde pública, enquadrando o país como um dos epicentros de controle epidemiológico da coinfecção. Tal fato reflete os fatores socioeconômicos, demográficos e clínicos, caracterizando a prevalência da enfermidade nas diversas regiões do país (CAVALIN RF, et al., 2020).

Nos indivíduos, a coinfecção TB-HIV implica na acelerada progressão de ambas as doenças e maior risco de falência no tratamento e desenvolvimento de multirresistência aos tuberculostáticos. Além de acarretar manifestações clínicas significativas, como confusão mental, hepatotoxicidade e nefrotoxicidade, a coinfecção ocasiona modificações nos âmbitos sexual, laboral, social e comportamental, impactando na qualidade de vida dos indivíduos (SILVA JDP, et al., 2020). 
De acordo com o Ministério da Saúde (MS), em indivíduos com TB, ações de diagnóstico precoce da infecção do HIV são prioritárias. A identificação prematura da coinfecção e o seu tratamento correto podem auxiliar na redução das taxas de: incidência, mortalidade, prevalência da coinfecção e os fatores associados, facilitando a detecção de pacientes em risco e efetividade das intervenções de controle (BARBOSA KMB, et al., 2020). Contudo, disparidades geográficas existentes no país, entre 2000 e 2011 em relação a mortalidade pela coinfecção, identificaram as regiões Norte, Nordeste e Centro-Oeste como de maior vulnerabilidade (BALDAN SS, et al., 2017).

Essa realidade também se faz presente em diversas nações, sobretudo, em regiões que são afetadas pela falta de recursos para prevenção, diagnóstico, tratamento e controle tanto do HIV quanto da TB (BARBOSA $\mathrm{KMB}$, et al., 2020). Além disso, a subnotificação dos casos confirmados representa um desafio para investigação da coinfecção e reconhecimento da população em risco, podendo representar a resistência dos indivíduos em reconhecer o desenvolvimento da doença ou, até mesmo, as falhas de comunicação entre os serviços de atenção à saúde e a população (BALDAN SS, et al., 2017).

Diante desse contexto, a notificação epidemiológica da coinfecção TB-HIV funciona como um importante indicador da qualidade dos serviços de saúde, das áreas de maior vulnerabilidade e dos grupos de risco, permitindo maior reflexão e aprimoramento das políticas e ações de manejo para alcançar as demandas e desafios da saúde no Brasil. Nesse sentido, o presente estudo objetivou analisar a distribuição temporal e epidemiológica da coinfecção TB-HIV no Estado do Pará, no período de 2010-2020.

\section{MÉTODOS}

Trata-se de um estudo ecológico, retrospectivo e descritivo, de séries temporais, dos casos confirmados de TB coinfectados com HIV obtidos no Sistema de Informação de Agravos de Notificação (SINAN), da plataforma do Departamento de Informática do Sistema Único de Saúde (DATASUS), segundo as características sociodemográficas e clínicas.

O local de estudo foi o estado do Pará, situado no Norte do Brasil, e seus 144 municípios, que possui uma população estimada em 2021 de 8.777.124 habitantes e densidade demográfica de 6,07 hab/km² (IBGE, 2011; IBGE, 2021). Foram incluídos todos os casos de TB coinfectados pelo HIV com diagnóstico confirmado no estado paraense no período de 2010 a 2020.

As variáveis sociodemográficas estudadas foram: ano da notificação; município; sexo; faixa etária e escolaridade. Variáveis clínicas: forma clínica; uso de antirretroviral; situação de encerramento e critério de confirmação. Além disso, foram coletadas informações sobre doenças e agravos associados (diabetes mellitus, tabagismo, alcoolismo, doença mental e drogas ilícitas) e três grupos vulneráveis (população privada de liberdade, população em situação de rua e profissionais de saúde) para TB-HIV.

A análise da coinfecção TB-HIV foi realizada entre os casos confirmados de TB com sorologia anti-HIV positiva confirmados no período de 2010 a 2020. Os dados foram organizados em banco de dados e analisados a partir de estatística descritiva por meio das frequências relativa e absoluta, utilizando os programas Microsoft Office Excel 2019 e Minitab® versão 19.

O presente estudo, por ter trabalhado com dados de domínio público do DATASUS, disponíveis on-line, sem identificação pessoal ou institucional, não necessitou de aprovação do Comitê de Ética de Pesquisa, com base na Resolução № 510, de 7 de abril de 2016, do Conselho Nacional de Saúde.

\section{RESULTADOS E DISCUSSÃO}

No período de 2010 a 2020, 48.684 casos de TB foram registrados. Dentre estes, 4.307 apresentaram sorologia anti-HIV positiva, caracterizando uma coinfecção TB-HIV de $8,8 \%$ no estado do Pará no período estudado. A partir da análise temporal foi possível detectar uma tendência crescente dos casos de coinfecção TB-HIV, com percentual de 7,2\% em 2010 a 10,7\% em 2020. Este comportamento crescente foi observado a partir de 2012 e perdurou até 2017. O ano de 2019 se caracterizou como o de maior número de casos da coinfecção TB-HIV, correspondendo a $12,6 \%$ dos casos no período analisado (Figura 1). 
Figura 1 - Série temporal dos casos de coinfecção TB-HIV segundo ano diagnóstico no estado do Pará, 2010-2020. $(n=4.307)$

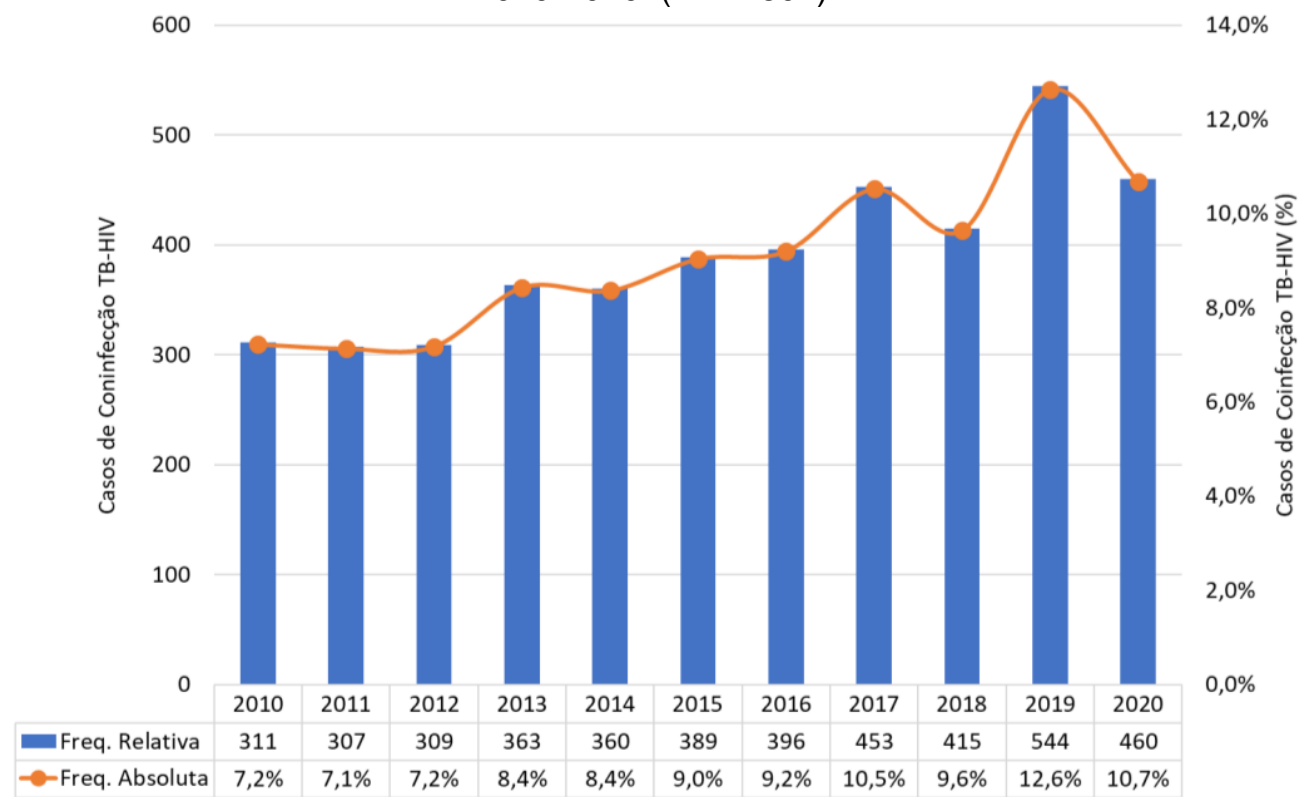

Fonte: Silva TC, et al., 2021; dados extraídos do SINAN, 2021.

Os dados mostraram aumento da coinfecção ao longo dos anos e ligeiro declínio ao final da série temporal, reforçando a necessidade do controle eficiente desta coinfecção. Este aumento também foi evidenciado em estudos realizados por Gaspar RS, et al. (2016) nos estados do Brasil e por Marques CC, et al. (2019) no Rio Grande do Norte. MS só reconheceu a importância da coinfecção TB-HIV em 2011 devido sua alta taxa de prevalência e óbitos em pacientes com TB, o que justifica o aumento das notificações a partir de 2012 (MINISTÉRIO DA SAÚDE, 2011; GASPAR RS, et al., 2016).

Quanto aos casos de coinfecção nos municípios, identificou-se que os pertencentes à Região Metropolitana de Belém (RMB) apresentaram os maiores percentuais, com proporção total de $45,5 \%(n=$ 22.134) dos casos, estando Belém, com 66,4\% ( $n=1.825)$, seguido por Ananindeua e Marituba, com 15,7\% $(n=431)$ e $4,3 \%(n=117)$, respectivamente. Dois municípios do sudeste paraense registraram os menores percentuais: Paragominas $(2,4 \%, n=65)$ e Parauapebas $(2,9 \%, n=81)$ (Figura 2). Diante da perspectiva apresentada, foi identificada alta coinfecção por TB-HIV no período analisado.

Figura 2 - Distribuição dos casos de coinfecção TB-HIV segundo os principais municípios do estado do Pará, 2010-2020. $(n=4.307)$

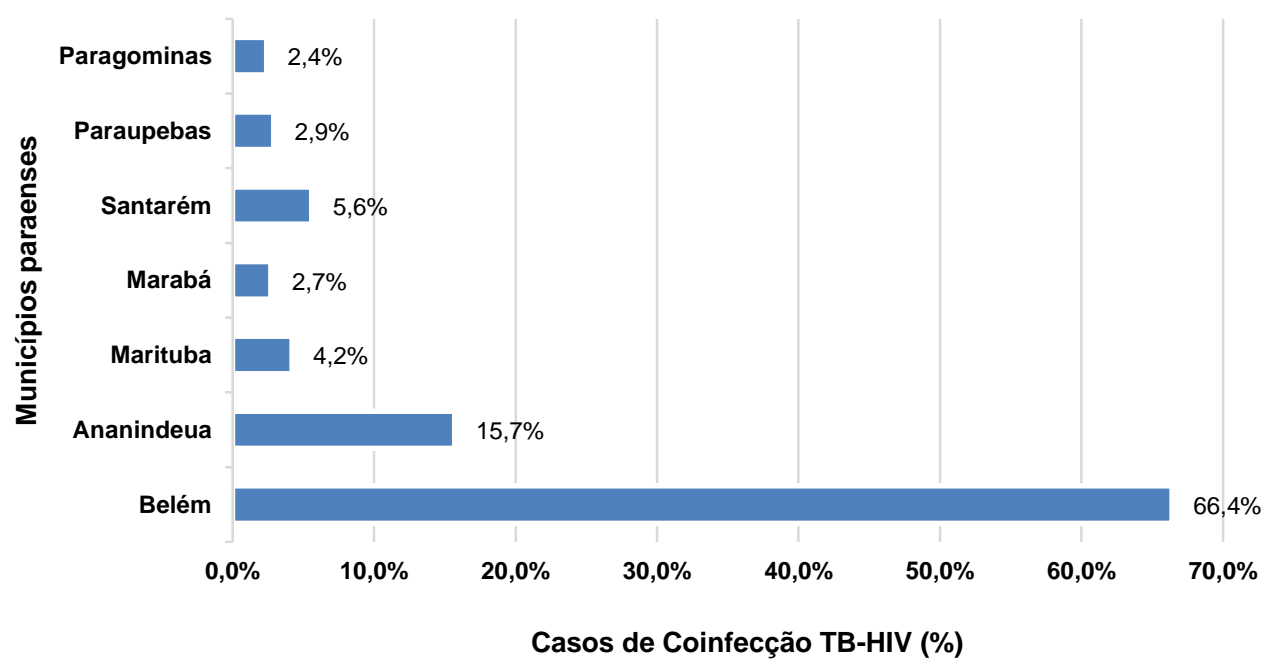

Fonte: Silva TC, et al., 2021; SINAN, 2021. 
Esses achados contribuem com o desenvolvimento de estratégias de controle da coinfecção nos municípios paraenses e, também, com a redução de morbimortalidade, manutenção da sexualidade e envelhecimento da população (MINISTÉRIO DA SAÚDE, 2021). Neste estudo, pode-se observar que o município de Belém se destacou com os maiores percentuais da coinfecção, devendo as medidas de controle e prevenção serem implementadas com maior efetividade.

Em relação às características sociodemográficas, foi observado a predominância de casos no sexo masculino $(70,3 \%)$ e faixa etária entre 20 a 39 anos $(57,0 \%, n=2.334)$, seguido de indivíduos entre 40 a 59 anos $(35,4 \%, n=1.448)$. Com base no nível de escolaridade, a maior ocorrência de coinfectados foi verificada em pessoas com ensino médio completo, correspondendo a 41,9\% $(n=629)$ dos casos (Tabela 1).

Tabela 1 - Caracterização sociodemográfica dos casos confirmados de coinfecção TB-HIV no estado do Pará, 2010-2020. $(n=4.307)$

\begin{tabular}{lcc}
\hline \multirow{2}{*}{ Variável } & N & Coinfecção TB-HIV \\
\cline { 2 - 3 } & & \\
\hline Sexo & 3.030 & 70,3 \\
\hline Masculino & 1.277 & 29,7 \\
Feminino & & 1,3 \\
\hline Faixa etária (anos) & 55 & 1,7 \\
\hline$<1$ & 69 & 57,0 \\
$15-19$ & 2.334 & 35,4 \\
$20-39$ & 1.448 & 4,6 \\
$40-59$ & 198 & \\
$\geq 60$ & & 23,5 \\
\hline Escolaridade & 353 & 41,9 \\
\hline Ens. Fundamental completo & 629 & 20,8 \\
Ens. Médio completo & 312 & 8,4 \\
Ens. Médio incompleto & 126 & 5,4 \\
Ens. Superior completo & 81 & $\mathbf{1 0 0 , 0}$ \\
Ens. Superior incompleto & $\mathbf{4 . 3 0 7}$ &
\end{tabular}

Fonte: Silva TC, et al., 2021; dados extraídos do SINAN, 2021.

O perfil sociodemográfico evidenciou maior ocorrência de coinfecção no sexo masculino, similar aos estudos realizados no estado de São Paulo (CAVALIN RF, et al., 2020; BRUNELLO MEF, et al., 2011). Isto pode está relacionado ao retardamento do diagnóstico de TB em pacientes com HIV e, principalmente, ao acesso limitado dos homens aos serviços de saúde (JÚNIOR ACV, et al., 2020). A predominância da coinfecção TB-HIV em indivíduos na faixa etária entre 20 a 39 anos pode estar relacionada ao estilo de vida e comportamentos vulneráveis dessa faixa etária, resultando em maior exposição (MAGNO ES, et al., 2017).

Durante a análise dos dados, observou-se que a maioria dos indivíduos possuem nível médio completo $(41,9 \%)$, resultado diferente do encontrado em um estudo ecológico realizado no Mato Grosso do Sul, o qual identificou que as pessoas mais acometidas pela doença possuíam baixa escolaridade. Ressalta-se que o nível de escolaridade representa como o acesso à educação é um fator que influencia a saúde da população, uma vez que demonstra a capacidade ou dificuldade do indivíduo em adquirir informações de promoção de saúde e prevenção de doenças, sendo caracterizado como um importante determinante da saúde (JÚNIOR ACV, et al., 2020).

Foi verificado que $68,3 \%$ dos indivíduos $(n=2.945)$ apresentam a forma clínica pulmonar. Em relação ao tipo de entrada, $78,6 \%(n=3.338)$ foram casos novos e $8,6 \%(n=373)$ identificados como reingressos após abandono. Quanto ao uso da Terapia Antirretroviral (TARV), 31,9\% $(n=1.378)$ usava a TARV e 58,6\% $(n=$ 2.528) permaneceu ignorado/branco. Analisando a situação de encerramento, $47,7 \%$ ( $n=2.059)$ evoluíram para a cura e $14,9 \%(n=645)$ para óbito por outras causas (Tabela 2). 
Tabela 2 - Distribuição das características clínicas na coinfecção de TB-HIV no estado do Pará, 2010-2020. $\underline{(n=4.307)}$

\begin{tabular}{|c|c|c|}
\hline \multirow{2}{*}{ Variável } & \multicolumn{2}{|c|}{ Coinfecção TB-HIV } \\
\hline & $\mathbf{N}$ & $\%$ \\
\hline \multicolumn{3}{|l|}{ Forma clínica } \\
\hline Pulmonar & 2.945 & 68,3 \\
\hline Extrapulmonar & 908 & 21,0 \\
\hline Pulmonar + Extrapulmonar & 454 & 10,5 \\
\hline \multicolumn{3}{|l|}{ Tipo de entrada } \\
\hline Casos novos & 3.388 & 78,6 \\
\hline Recidiva & 269 & 21,0 \\
\hline Reingresso pós-abandono & 373 & 8,6 \\
\hline Transferência & 257 & 5,9 \\
\hline Pós-óbito & 12 & 0,2 \\
\hline Não informado & 8 & 0,1 \\
\hline \multicolumn{3}{|l|}{ Uso de Antirretroviral } \\
\hline Sim & 1.378 & 31,9 \\
\hline Não & 401 & 9,3 \\
\hline Ing./Branco & 2.528 & 58,6 \\
\hline \multicolumn{3}{|l|}{ Situação de encerramento } \\
\hline Cura & 2.056 & 47,7 \\
\hline Abandono & 582 & 13,4 \\
\hline Mudança de esquema & 41 & 0,9 \\
\hline Transferência & 338 & 7,8 \\
\hline Óbito por Tuberculose & 142 & 3,2 \\
\hline Óbito por outras causas & 645 & 14,9 \\
\hline Ing./Branco & 456 & 10,7 \\
\hline Outros & 38 & 0,8 \\
\hline Total & 4.307 & 100,0 \\
\hline
\end{tabular}

Fonte: Silva TC, et al., 2021; dados extraídos do SINAN, 2021.

A forma pulmonar é a mais frequente e principal fonte infectante de disseminação da doença, se caracterizando como a mais relevante para a saúde pública, corroborando com os achados do presente estudo (SILVA JDP, et al., 2020). Quanto ao tipo de entrada, o Pará obteve um alto percentual de casos novos (78,6\%) quando comparado com estudo realizado no Mato Grosso do Sul, o qual identificou 69,5\% (proporção cerca de $9 \%$ menor). Tais índices evidenciam a importância do diagnóstico precoce de TB em pessoas com HIV, visando reduzir a multiplicação do bacilo e a probabilidade de formação de cavitações pulmonares (forma mais eficaz de propagação do bacilo e de maior gravidade), podendo ter maiores chances de sobrevida (BALDAN SS, et al., 2017).

Ressalta-se que o MS preconiza intervenções para controle da coinfecção TB-HIV, destacando a testagem oportuna para HIV para todos os portadores de TB por meio do teste rápido. Também recomenda como diretriz nacional, o diagnóstico precoce, tratamento da TB ativa e latente, bem como o início oportuno da TARV (MINISTÉRIO DA SAÚDE, 2019a).

Em relação ao uso da TARV, os resultados mostraram maior proporção de ignorados/branco (58,6\%). Isso sugere a importância de aprimorar os sistemas de preenchimento dos dados presentes nas fichas de notificação, objetivando minimizar possíveis erros e interpretações da situação de saúde à nível nacional. Segundo Coelho LE, et al. (2016), a TARV reduz entre $44 \%$ a $72 \%$ a mortalidade de indivíduos infectados pelo HIV durante o tratamento da TB. No entanto, estes autores afirmam que o consumo de antirretrovirais se torna desafiador por conta da adesão de múltiplas drogas e a justaposição da toxicidade dos tuberculostáticos e dos antirretrovirais, aumentando a interrupção do tratamento e/ou à necessidade de esquemas alternativos. 
Sabe-se que o MS estabelece uma taxa de cura de $85,0 \%$ para a TB e abandono inferior a 5,0\%. Os resultados obtidos demonstraram uma taxa de cura dos casos da coinfecção TB-HIV no estado do Pará $(47,4 \%)$ abaixo do recomendado, assim como para as taxas de abandono de tratamento que permaneceu, no presente estudo, com 10,8\%. Em relação ao óbito por TB, foi verificado no Pará 3,2\% de casos no período estudado, sendo bem menor que os resultados de estudo realizado no estado de São Paulo, com o índice $32,2 \%$ óbitos por decorrência da TB (CAVALIN RF, et al., 2020).

A coinfecção TB-HIV aumenta o número de mortes dos indivíduos acometidos por ambas as infecções quando não há adesão ao tratamento de TB. Esta alta proporção de mortes indica um sistema de saúde ineficiente tanto no diagnóstico de TB e HIV quanto no início da TARV, além de representar falta de acesso do indivíduo aos serviços de saúde, ações e cuidados, visto que a TB é uma doença com tratamento reconhecido e gratuito no Brasil, devendo ser iniciado desde que ocorra um diagnóstico precoce e adequado (MAGNABOSCO GT, et al., 2016).

No presente estudo, quanto aos fatores associados à coinfecção TB-HIV, verificou-se que $16,8 \%$ ( $n=722)$ havia dependência ao álcool e 9,4\% $(n=403)$ eram tabagistas e $8,7(n=376)$ tinha o consumo de drogas ilícitas (Tabela 3).

Tabela 3 - Levantamento dos principais fatores de risco na coinfecção de TB-HIV no Estado do Pará, 20102020.

\begin{tabular}{lcccccc}
\hline \multirow{2}{*}{ Fatores de Risco } & \multicolumn{2}{c}{ Sim } & \multicolumn{2}{c}{ Não } & \multicolumn{2}{c}{ Ing./ Branco } \\
\cline { 2 - 7 } & $\mathbf{N}$ & $\%$ & $\mathbf{N}$ & $\%$ & $\mathbf{N}$ & $\%$ \\
\hline Diabético & 124 & 2,9 & 3623 & 84,1 & 560 & 13,0 \\
Tabagismo & 403 & 9,4 & 2027 & 47,1 & 1877 & 43,6 \\
Alcoolismo & 722 & 16,8 & 3003 & 69,7 & 582 & 13,5 \\
Doença mental & 80 & 1,9 & 3655 & 84,9 & 572 & 13,3 \\
Doenças ilícitas & 376 & 8,7 & 2043 & 47,4 & 1888 & 43,8 \\
\hline
\end{tabular}

Fonte: Silva TC, et al., 2021; dados extraídos do SINAN, 2021.

Com base nos fatores de risco, indivíduos com Diabetes Mellitus (DM), doenças mentais e com uso de drogas, tabaco e álcool possuem maior propensão a adoecer por TB (SILVA DR, et al., 2018). O consumo de drogas ilícitas, álcool e tabaco associado à TB em pessoas portadoras de HIV, cria uma vulnerabilidade social que dificulta o controle da doença (OLIVEIRA RL, et al., 2020).

As pessoas que fazem uso frequente de drogas ilícitas favorecem a transmissão da TB pulmonar através do compartilhamento de seringas e agulhas; além da interação nociva destas drogas com o organismo, dificultando na capacidade de compreensão sobre a importância do tratamento e, também, na atuação dos profissionais de saúde (OLIVEIRA RL, et al., 2020; JUSTO MT, et al., 2018).

Em estudo realizado em Campo Grande, Mato Grosso do Sul, 27\% dos coinfectados por TB-HIV eram tabagistas (CUNHA JPA, et al., 2021), enquanto que, no presente estudo, apenas 9,4\% eram tabagistas (Tabela 3). Entretanto, análises sistemáticas e meta-análises de estudos observacionais mostraram associação desfavorável entre a epidemia global de TB e tabagismo, sendo a exposição ao tabagismo associada à infecção por TB, TB ativa e mortalidade relacionada à TB (BATES MN, et al., 2007; SILVA DR, et al., 2018).

Em relação aos casos de coinfecção TB-HIV em grupos vulneráveis, os resultados evidenciaram que, dos 4.307 casos que apresentaram sorologia anti-HIV positiva, 233 eram de grupos vulneráveis: População Privada de Liberdade (PPL), População em Situação de Rua (PSR) e profissionais de saúde, correspondendo a 5,4\% dos casos de coinfecção TB-HIV (Figura 3). As circunstâncias as quais estão inseridos, bem como as condições socioeconômicas, configuram-se como um fator determinante para o adoecimento em comparação à população geral (BLACK TPL e CARDOSO MD, 2018). 
Figura 3 - Distribuição dos casos de coinfecção TB-HIV de acordo com os grupos vulneráveis e ano diagnóstico no estado do Pará, 2010-2020.

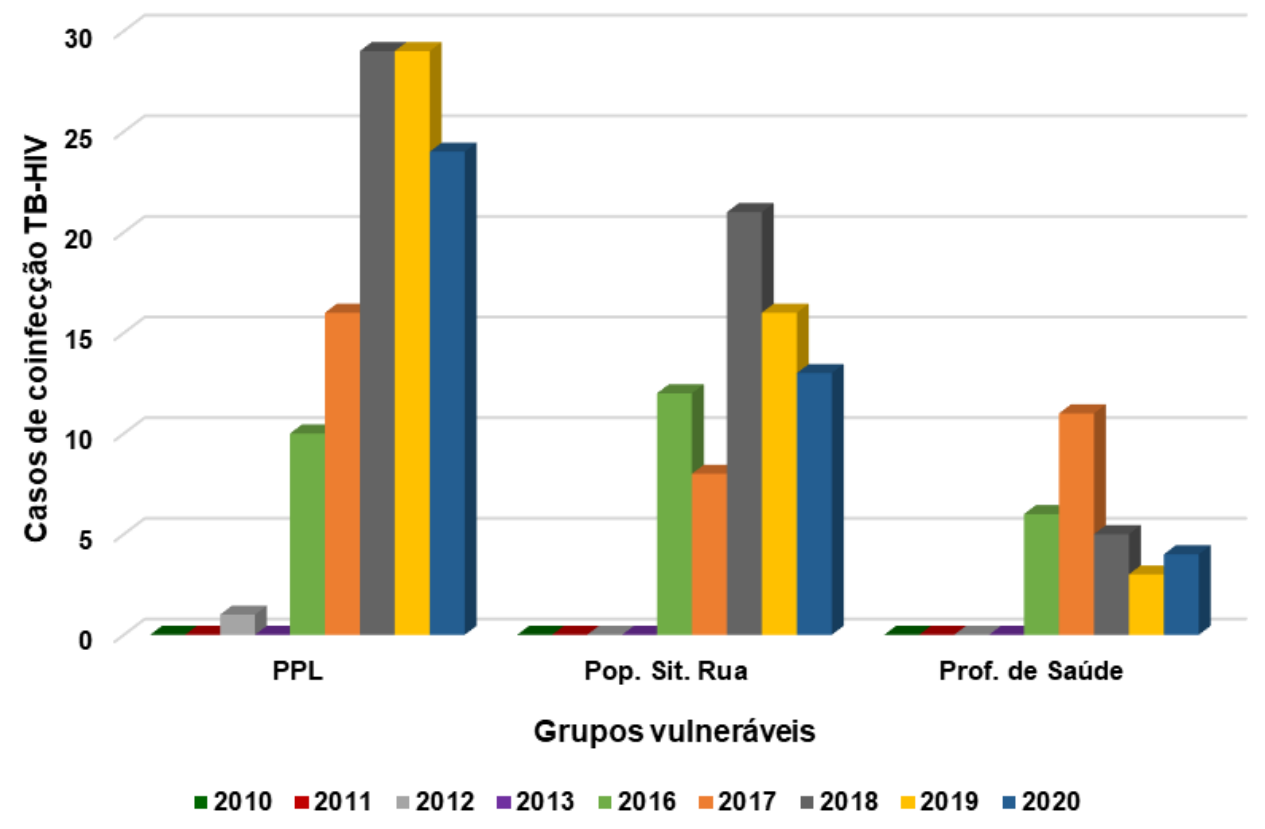

Fonte: Silva TC, et al., 2022; dados extraídos do SINAN, 2021.

Diante dos casos de coinfecção TB-HIV, foram confirmados 119 casos correspondente a PPL no estado do Pará no período estudado, identificando uma alta ocorrência de coinfecção nos anos de 2018 e 2019 , equivalente a $48,7 \%$ em relação aos outros anos. As altas taxas de encarceramento, superlotação e ausência de assistência à saúde são fatores determinantes que contribuem para o elevado coeficiente de adoecimento no sistema prisional, corroborando com o estudo de Medeiros JC, et al. (2012) que afirmam sobre a maior vulnerabilidade desse grupo pela exposição aos fatores de riscos.

Em relação aos casos de coinfecção TB-HIV na PSR, o ano de 2018 apresentou maior número de casos $(n=21)$. A PSR é uma das populações mais vulneráveis em relação à coinfecção TB-HIV, permeando um maior grau de letalidade devido o comprometimento imunológico, elevado risco de adoecimento pelo estilo de vida, comportamentos arriscados, pobreza e o uso de substâncias ilícitas, com associação da vulnerabilidade das políticas de comunicação e falta de acesso às instituições e órgãos de saúde (MINISTÉRIO DA SAÚDE, 2019b; ORFÃO NH, et al., 2021).

Os profissionais da saúde apesar de representarem 14,2\% casos neste estudo, se enquadram como um grupo de vulnerabilidade devido ao convívio com as pessoas acometidas por TB e/ou HIV e o importante papel que assumem no controle, prevenção e tratamento. Dentre as especialidades mais expostas à coinfecção, enfermeiros e médicos se destacam por atuarem diretamente com pacientes coinfectados (SANTOS JLG, et al., 2012).

Além disso, outros aspectos que podem levar ao acometimento de tais profissionais, no que tange a TB, são o uso inadequado de equipamentos de proteção individual, pois sabe-se que o bacilo apresenta um alto grau de transmissibilidade por aerossóis e, no HIV, uma das principais causas de transmissão em profissionais da saúde são os acidentes com materiais perfurocortantes contaminados ((ARAÚJO MR, et al., 2016; SOUZA MCM e FREITAS MI, 2010).

Neste contexto, como medida do segundo pilar da Estratégia de Controle da TB, o Brasil recomenda ações de proteção social e acesso aos serviços de saúde, sendo importante identificar o comportamento da infecção nestes grupos vulneráveis (MACEDO LR, et al., 2021). Logo, diferentes estratégias devem ser traçadas para as populações vulneráveis, sendo imprescindível para as PPL, PSR e profissionais de saúde políticas públicas específicas para prevenção e controle da coinfecção TB-HIV (CAVALIN RF, et al., 2020). 
É importante enfatizar que estudos ecológicos possuem algumas limitações próprias dos estudos que utilizam dados secundários extraídos de sistemas de informações de saúde, como conter incompletudes nos registros e desatualização dos dados, o que dificulta a real compreensão da situação local e a caracterização de alguns casos, logo, as análises devem ser observadas com prudência (MAGNO ES, et al., 2017).

Contudo, do ponto de vista da vigilância epidemiológica, a notificação da coinfecção é um quadro complexo e tem como objetivo analisar a vulnerabilidade individual de cada grupo selecionado. É através dessa análise que são identificados os indicadores para gerenciamento das endemias e formulação de intervenções apropriadas para a coletividade, visando a redução da morbimortalidade da coinfecção das duas doenças (MEDEIROS CJ, et al., 2012). Dessa maneira, apesar das limitações, os dados secundários permitem conhecer o diagnóstico de saúde de grupos específicos (MARQUES CC, et al., 2019).

\section{CONCLUSÃO}

A análise dos casos de coinfecção TB-HIV no Estado do Pará no período de 2010 a 2020, identificou um aumento ao longo dos anos, com maior ocorrência no ano de 2019. A capital do estado, Belém, foi o município mais acometido pela coinfecção. As características epidemiológicas demonstraram maior número de coinfectados do sexo masculino, adultos jovens, com ensino médio completo e, as clínicas, maior percentual da forma pulmonar, casos novos e cura dos casos. Além disso, foi evidenciado que a maioria dos indivíduos não possuíam influência de outros fatores de risco e, em relação aos grupos vulneráveis, a população privada de liberdade foi a mais acometida. Os resultados presentes neste estudo fornecem informações epidemiológicas e ao longo do tempo sobre a coinfecção TB-HIV relevantes para futuras pesquisas e, também, favorecem o aperfeiçoamento e implementação de estratégias de prevenção, diagnóstico precoce, controle e tratamento, visando melhores condições de saúde e qualidade de vida aos indivíduos acometidos pela coinfecção.

\section{REFERÊNCIAS}

1. ARAÚJO MR, et al. Avaliação situacional de biossegurança em tuberculose em Unidades Básicas de Saúde na Amazônia. Revista Brasileira de Saúde Ocupacional, 2016; 41(e21): 1-9.

2. BALDAN SS, et al. Características clínico-epidemiológicas da coinfecção por tuberculose e HIV e sua relação com o Índice de Desenvolvimento Humano no estado do Mato Grosso do Sul, Brasil. Revista Pan-Amazônica de Saúde, 2017; 8(3): 59-67.

3. BARBOSA KMB, et al. Análise da associação da coinfecção TB/HIV com sexo e forma clínica da TB no Piauí. 1a. ed. São Paulo: Científica Digital, 2020; 15-24p.

4. BATES MN, et al. Risk of tuberculosis from exposure to tobacco smoke: a systematic review and meta-analysis. Archives of internal medicine, 2007; 167(4): 335-342.

5. BLACK TLP, CARDOSO MD. Coinfecção TB/HIV em adolescentes residentes em Pernambuco, notificados no período de 2001 a 2016. Brazilian Journal of Health Review, 2019; 2(1): 321-334.

6. BRUNELLO MEF, et al. Áreas de vulnerabilidade para co-infecção HIV-aids/TB em Ribeirão Preto, SP. Revista de Saúde Pública, 2011;45(3):556-63.

7. CAVALIN RF, et al. TB-HIV co-infection: spatial and temporal distribution in the largest Brazilian metropolis. Revista de Saúde Pública, 2020;54: 112.

8. COELHO LE, et al. O tratamento da coinfecção HIV-TB. The Brazilian Journal of Infectious Diseases, 2016;2(5): 134148.

9. CUNHA JPA, et al. Fatores de risco e comorbidades associados aos casos de tuberculose notificados no município de Campo Grande, Mato Grosso do Sul. Revista Eletrônica Acervo Saúde, 2021;13(8): e8676.

10. GASPAR RS, et al. Temporal analysis of reported cases of tuberculosis and of tuberculosis-HIV co-infection in Brazil between 2002 and 2012. Jornal Brasileiro de Pneumologia, 2016;42(6): 416-422.

11. INSTITUTO BRASILEIRO DE GEOGRAFIA E ESTATíSTICA (IBGE). Censo Demográfico 2010, Área territorial brasileira. Rio de Janeiro: IBGE, 2011. Disponível em: https://cidades.ibge.gov.br/brasil/pa/panorama. Acessado em: 10 de janeiro de 2022.

12. INSTITUTO BRASILEIRO DE GEOGRAFIA E ESTATíSTICA (IBGE). Diretoria de Pesquisas, Coordenação de População e Indicadores Sociais, Estimativas da população residente com data de referência 10 de julho de 2020. IBGE, 2021. Disponível em: https://cidades.ibge.gov.br/brasil/pa/panorama. Acessado em: 10 de janeiro de 2022. 
13. JÚNIOR ACV, et al. Avaliação do perfil epidemiológico da tuberculose e a sua coinfecção TBHIV nos estados da Paraíba e Rio Grande do Norte. Brazilian Journal of Development, 2020;6(1): 441-456.

14. JUSTO MT, et al. Associação entre tuberculose e consumo de drogas lícitas e ilícitas. Enfermagem Brasil, 2018;17(5): 460-470.

15. MACEDO LR, et al. Populações vulneráveis e o desfecho dos casos de tuberculose no Brasil. Ciência \& Saúde Coletiva, 2021;26(10): 4749-4759.

16. MAGNABOSCO GT, et al. Controle da Tuberculose em pessoas vivendo com HIV/aids. Revista Latino-Americana de Enfermagem, 2016; 24:e2798.

17. MAGNO ES, et al. Fatores associados à coinfecção tuberculose e HIV: o que apontam os dados de notificação do Estado do Amazonas, Brasil, 2001-2012. Cadernos de Saúde Pública, 2017; 33(5):e00019315.

18. MARQUES CC, et al. Casos de tuberculosis coinfectados por VIH en el estado del noreste brasileño. Revista Enfermería Actual, 2019;36.

19. MEDEIROS CJ, et al. Características demográficas e clínicas dos casos de tuberculose notificados pelo Núcleo de Epidemiologia Hospitalar no Município de Vitória, Estado do Espírito Santo, Brasil,. Epidemiologia e Serviços de Saúde, 2012;21(1): 159-166.

20. MINISTÉRIO DA SAÚDE. Manual de recomendações para o controle da tuberculose no Brasil. 2019a. Disponível em: http://www.aids.gov.br/pt-br/pub/2019/manual-de-recomendacoes-para-o-controle-da-tuberculose-no-brasil. Acessado em: 8 de janeiro de 2022.

21. MINISTÉRIO DA SAÚDE. Panorama epidemiológico da coinfecção TB-HIV no Brasil 2020. 2021. Disponível em: http://www.aids.gov.br/pt-br/pub/2021/panorama-epidemiologico-da-coinfeccao-tb-hiv-no-brasil-2020. Acessado em: 14 de dezembro de 2021.

22. MINISTÉRIO DA SAÚDE. Panorama epidemiológico da coinfecção TB-HIV no Brasil 2019. 2019b. Disponível em: https://prceu.usp.br/wp-content/uploads/2020/10/2019-Ministerio-da-Saude-boletim_tuberculose_-HIV.pdf. Acessado em: 20 de janeiro de 2022.

23. OLIVEIRA RL, et al. Relatos de uso de tabaco, álcool e drogas ilícitas entre pacientes em tratamento para tuberculose. Brazilian Journal of Health Review, 2020; 3(5): 14866-14877.

24. ORFÃO NH, et al. População em situação de rua: perfil dos casos de coinfecção tuberculose e HIV. Revista Enfermagem Contemporânea, 2021;10(1): 94-102.

25. SANTOS JLG, et al. Risco e vulnerabilidade nas práticas dos profissionais de saúde. Revista Gaúcha de Enfermagem, 2012;33(2): 205-212.

26. SILVA DR, et al. Fatores de risco para tuberculose: diabetes, tabagismo, álcool e uso de outras drogas. Jornal Brasileiro de Pneumologia, 2018;44(2): 145-152.

27. SILVA JDP, et al. Tendência temporal da incidência da coinfecção TB/HIV e testagem de HIV da população idosa brasileira de 2008 a 2018. Revista Brasileira de Geriatria e Gerontologia, 2020;23(3): e200215.

28. SOUZA MCM, FREITAS MI. Representações de profissionais da atenção primária sobre risco ocupacional de infecção pelo HIV. Revista Latino-Americana de Enfermagem, 2010;18(4): 1-8. 